\title{
The human papilloma virus (HPV)-18 E6 oncoprotein physically associates with Tyk 2 and impairs Jak-STAT activation by interferon- $\alpha$
}

\author{
Suiyang Li ${ }^{1}$, Sylvie Labrecque ${ }^{5,6}$, M Cristina Gauzzi ${ }^{7}$, Andrew R Cuddihy ${ }^{1}$, Andrew HT Wong ${ }^{1}$, \\ Sandra Pellegrini ${ }^{7}$, Gregory J Matlashewski ${ }^{5,6}$ and Antonis E Koromilas ${ }^{*, 1,2,3,4}$ \\ ${ }^{1}$ Department of Oncology, McGill University, Montreal, Quebec, Canada; ${ }^{2}$ Department of Medicine, McGill University, Montreal, \\ Quebec, Canada; ${ }^{3}$ Department of Microbiology and Immunology, McGill University, Montreal, Quebec, Canada; ${ }^{4}$ Department of \\ Anatomy and Cell Biology, McGill University, Montreal, Quebec, Canada; ${ }^{5}$ Institute of Parasitology, McGill University, Montreal, \\ Quebec, Canada; ${ }^{6}$ McGill Cancer Center, McGill University, Montreal, Quebec, Canada; ${ }^{7}$ Pasteur Institute, INSERM U 276. \\ Paris 75724 Cedex 15, France
}

\begin{abstract}
We have examined the effects of human papilloma virus (HPV) E6 proteins on interferon (IFN) signaling. Here we show that expression of the 'malignant' HPV-18 E6 in human HT1080 cells results in inhibition of JakSTAT activation in response to IFN- $\alpha$ but not IFN- $\gamma$. This inhibitory effect is not shared by the 'benign' HPV11 E6. The DNA-binding and transactivation capacities of the transcription factor ISGF3 are diminished in cells expressing HPV-18 E6 after IFN- $\alpha$ treatment as a result of decreased tyrosine phosphorylation of Tyk2, STAT2 and STAT1. However, HPV-18 E6 does not affect the induction of tyrosine phosphorylation and DNA-binding of STAT1 by IFN- $\gamma$. In addition, HPV E6 proteins physically interact with Tyk2. This interaction takes place preferably with HPV-18 E6 and to a lesser extent with HPV-11 E6. The E6/Tyk2 interaction requires the $\mathrm{JH}_{6}-\mathrm{JH}_{7}$ domains of $\mathrm{Tyk2}$, which are important for Tyk2 binding to the cytoplasmic portion of IFN- $\alpha$ receptor 1 (IFNAR1). These findings demonstrate an inhibitory role of HPV-18 E6 in the IFN- $\alpha$-induced JakSTAT pathway, which may be explained, at least in part, by the ability of E6 to interact with and impair Tyk2 activation.
\end{abstract}

Keywords: interferon; human papilloma virus; cell signaling; oncoproteins; protein phosphorylation; DNA-binding

\section{Introduction}

In humans, HPV infections often result in the formation of a variety of warts, i.e. papillomas, on skin or at anogenital organs. Among the total of 77 HPV subtypes identified so far, HPV-16 and 18 are two subtypes that retain the strongest tumorigenic activity, and belong to the 'high risk' group of HPVs. Some of the HPV encoding proteins, i.e. E6, E7 and E5 from the 'high risk' HPVs have been shown to exhibit transforming activities (for review see Howley, 1996; zur Hausen, 1996). It has been demonstrated that E6 protein from the 'high risk' but not the 'low risk' HPVs

*Correspondence: AE Koromilas, Lady Davis Institute for Medical Research, Jewish General Hospital, 3755 Cote-Ste-Catherine Street, Montreal, Quebec, Canada H3T 1E2

Received 8 January 1999; revised 10 May 1999; accepted 10 May 1999 has the capacity to bind to the tumor suppressor p53 and promote its degradation through ubiquitinmediated proteolysis (Howley, 1996). Expression of HPV-16 E6 alone is able to immortalize human mammary epithelial cells, or induce anchorageindependent growth of NIH3T3 cells (Howley, 1996; zur Hausen, 1996). It has also been shown that HPV16 E6 inhibits the serum- and calcium-induced differentiation of human keratinocytes (Sherman and Schlegel, 1996). Despite all these functions, other roles of E6, particularly the ones from the 'high risk' HPVs, in HPV-associated diseases and malignant tumor formation still remain elusive.

Interferons (IFNs) are a family of cytokines that exhibit strong antiviral and antiproliferative activities (for review see Vilcek and Sen, 1996). The signal transduction pathway used by IFNs has become a prototype for signaling by many other cytokines (for review see Stark et al., 1998). The binding of IFNs to their receptors elicits multiple signal transduction pathways which lead to the activation of different sets of genes. A consensus cis-acting DNA sequence that confers inducibility by IFN- $\alpha / \beta$ is present in the $5^{\prime}$ flanking regions of all $\underline{\mathrm{IFN}}-\alpha / \beta$-stimulated genes (ISGs) examined so far. This sequence is termed the IFNstimulated response element (ISRE). Stimulation with type I IFN (i.e. IFN- $\alpha / \beta$ ) induces binding to ISRE of a factor known as IFN-stimulated gene factor-3 (ISGF3), which is required for the transcriptional activation of ISRE-containing genes. Active ISGF3 is composed of four functional subunits. Three of these proteins are called signal transducer and activators of transcription (STAT). They are 113 (STAT2), 91 (STAT $1 \alpha), 84$ (STAT1 $\beta) \mathrm{kDa}$ in size, collectively termed ISGF $3 \alpha$ proteins and reside in the cytoplasm of unstimulated cells as monomers. In cells stimulated with IFN- $\alpha / \beta$, ISGF3 $\alpha$ proteins are tyrosine-phosphorylated, multimerize, translocate to the nucleus and bind DNA in the presence of a fourth protein, ISGF3 $\gamma$, a $48 \mathrm{kDa}$ DNA-binding protein (Bluyssen et al., 1996), thereby forming the ISGF3 active complex. Tyrosine phosphorylation of ISGF $3 \alpha$ proteins is mediated by activated cytoplasmic tyrosine kinases of the Janus (JAK) family, Jak1 and Tyk2, which associate with the receptor cytoplasmic tails (for review see Pellegrini and Dusanter-Fourt, 1997). Activated Jaks become autophosphorylated and subsequently phosphorylate the receptor on specific tyrosine residues, which function as docking sites for the src homology 2 (SH2) domain of STATs. 
Similarly, type II IFN (i.e. IFN- $\gamma$ ) induces transcription through binding to the >IFN-gamma activation site (GAS) by activated STAT1 homodimers (for review see Darnell, 1997). A single phosphorylation site on STAT1 at tyrosine ${ }^{701}$, which is mediated through Jak1 and Jak2 (Pellegrini and Dusanter-Fourt, 1997), appears to be both necessary and sufficient to mediate nuclear translocation, DNAbinding activity, and transcriptional activation by IFN- $\gamma$. In addition to tyrosine phosphorylation, serine ${ }^{727}$ phosphorylation of STAT1 is also required for maximal transcriptional activation (Darnell, 1997; Kovarik et al., 1998).

The use of IFNs for the treatment of HPVassociated cervical intraepithelial neoplasia (CIN) or cervical cancer has been under clinical trials (for review see Rockley and Tyring, 1995; Shah and Howley, 1996). Despite the satisfactory results in some of the clinical studies, IFN treatment is still far from widespread therapy against HPV infections. The success of IFNs appears to be largely dependent on the type of HPV and the immune response of the patients. Warts that are caused by the 'low risk' HPV-6 or 11 have high response rates to IFN treatment, whereas those, which are caused by the 'high risk' HPV-16 or 18, do not (Stellato, 1992; Trofatter, 1991). Considering the significance of HPV-associated disease in the human population and the little knowledge about the nature of HPV resistance to the IFN treatment, we sought to examine a role of HPV-18 oncoproteins in IFN signaling. In this paper, we provide strong evidence that HPV-18 E6 functions as a negative regulator of the Jak-STAT pathway activated by IFN- $\alpha$ through the inhibition of Tyk 2 activation. We also show that Tyk2 physically associates with E6. The Tyk2/E6 interaction is stronger with HPV-18 E6 than HPV-11 E6 and requires the $\mathrm{JH}_{6}-\mathrm{JH}_{7}$ domains of Tyk2, which are necessary for Tyk2 binding to IFNAR1. These novel findings may reveal important mechanisms utilized by 'high risk' HPVs to counteract IFN treatment.

\section{Results}

Inhibition of ISGF-3 DNA-binding in response to IFN- $\alpha$

To investigate a possible role of HPV-18 E6 oncoprotein in the JAK-STAT signaling, we used the human fibrosarcoma epithelial-like HT1080 cells stably expressing the HPV-18 E6 (Labrecque and Matlashewski, 1995). HT1080 cells transfected with the neomycin resistant gene (control cells) and HT1080 cells expressing HPV-18 E6 were stimulated with IFN- $\alpha$ for various periods of time. DNA-binding of ISGF3 was analysed by electrophoretic mobility shift assays (EMSAs) using a dsDNA oligonucleotide, which encompasses the ISRE of the ISG-15 gene (Reich and Darnell, 1989). Formation of a DNA/ protein complex with the specificity and mobility of ISGF3 was induced in both HT1080 cell lines at 30, 60 and 90 min after IFN- $\alpha$ treatment (Figure 1A, top panel, lanes 2-4 and 7-9). ISGF3 DNA-binding was $\sim$ fivefold higher in control cells than in cells expressing HPV-18 E6 (compare lanes 2-4 with 7-
(A)

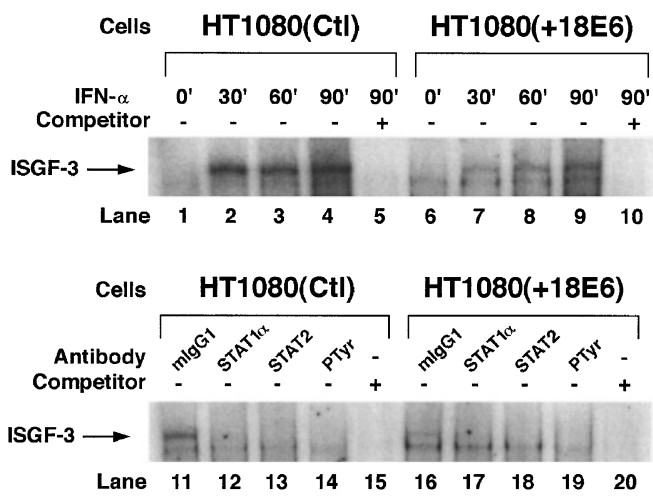

(B)

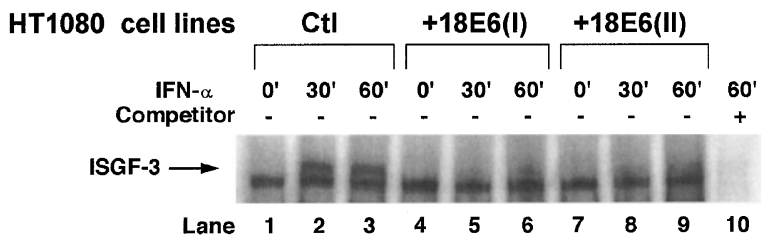

Figure 1 Inhibition of ISGF3 DNA-binding by HPV-18 E6. (A) HT1080 control cells (lanes $1-5$ and 11-15) and HT1080 cells expressing HPV-18 E6 (lanes 6-10 and 16-20) were treated with $1000 \mathrm{IU} / \mathrm{ml} \mathrm{IFN-} \alpha$ for 30 (lanes 2 and 7), 60 (lanes 3 and 8 ) and $90 \mathrm{~min}$ (lanes 4 and 9). Cell extracts were then analysed for DNA-binding using a ${ }^{32}$ P-labeled dsDNA ISRE oligonucleotide from ISG-15 gene promoter. A 100-excess of unlabeled dsDNA oligonucleotide was added in the cold competition reactions (lanes 5, 10, 15 and 20). For supershift assays, extracts from $30 \mathrm{~min}$ after IFN- $\alpha$ stimulation were preincubated with $2 \mu \mathrm{g}$ of mouse $\operatorname{IgG}_{1}$ (lanes 11 and 16), antiSTAT1 $\alpha$ (lanes 12 and 17), anti-STAT2 (lanes 13 and 18) and anti-PTyr (lanes 14 and 19) antibodies. (B) HT1080 control cells (lanes $1-3$ and 10) and two independent polyclonal populations of HT1080 cells expressing HPV-18 E6 (Pool I, lanes 4-6; Pool II, lanes 7-9) were stimulated with $1000 \mathrm{IU} / \mathrm{ml}$ of IFN- $\alpha$ for 30 (lanes 2, 5 and 8) and $60 \mathrm{~min}$ (lanes 3, 6, 9 and 10). ISGF3 DNA-binding was then examined using ${ }^{32} \mathrm{P}$-labeled ISRE from the ISG-15 gene promoter. A 100-fold excess of unlabeled ISRE oligonucleotide was added in the cold competition reaction in lane 10

9). To confirm the identity of the induced DNAprotein complex, antibodies to STAT-1 $\alpha$ (Figure 1A, bottom panel, lanes 12 and 17), STAT-2 (lanes 13 and 18) and phosphotyrosine (PTyr) (lanes 14 and 19) were added in the EMSA reactions. As a control for nonspecific binding, mouse $\operatorname{IgG}_{1}$ antibody was used (lanes 11 and 16). ISGF3 complex formation was abolished by the anti-STAT and anti-PTyr antibodies demonstrating that the induced DNA-binding complex consisted of tyrosine phosphorylated STAT1 $\alpha$ and STAT2.

To eliminate the possibility that differences in ISGF3 DNA-binding were due to clonal variations, we examined ISGF3 DNA-binding in two separate polyclonal populations of HT1080 cells transfected with the HPV-18 E6 gene. Similar to the E6 expressing HT1080 clone, ISGF3 DNA-binding by IFN- $\alpha$ was inhibited in both E6 polyclonal populations of HT1080 cells (Figure 1B, compare lane 2 with 5 and 8, lane 3 with 6 and 9) demonstrating that the effect of HPV-18 E6 was not clone specific. 
HPV-18 E6 inhibits STAT1 DNA-binding in response to $I F N-\alpha$ but not IFN- $\gamma$

Activation with IFN- $\alpha$ may also lead to STAT1 homodimerization and binding to the GAS DNA element of IFN-inducible genes (Pine et al., 1994). Based on this, we then examined STAT1 DNAbinding in cell extracts from IFN- $\alpha$-treated cells by EMSA using a dsDNA oligonucleotide that encompassed the GAS sequence of the promoter region of the IFP-53 gene (Strehlow et al., 1993). Induction of STAT1 DNA-binding was transient in both cell lines and was observed as early as $30 \mathrm{~min}$ after stimulation (Figure 2A, lanes 2 and 7). However, STAT1 DNAbinding was $\sim$ fivefold higher in control cells compared to HPV-18 E6 expressing cells (Figure 2A, compare lanes 2 and 7). Incubation of EMSA reactions with a monoclonal antibody to STAT1 $\alpha$ (lanes 12 and 17) or anti-PTyr (lanes 15 and 19) abolished DNA-binding. On the other hand, an antiSTAT2 monoclonal antibody (lanes 13 and 18) did not affect STAT1 DNA-binding suggesting that STAT $1 \alpha$ is most likely the only component of the DNA/protein complex.

Then we examined activation of STAT1 in response to IFN- $\gamma$. In control HT1080 cells induction of STAT1 DNA-binding was transient and reached the peak level at $30 \mathrm{~min}$ of IFN- $\gamma$ treatment but declined gradually at 60 and $90 \mathrm{~min}$ (Figure 2B, lanes 2-4). The same level of induction was observed in HT1080 cells expressing the HPV-18 E6 (Figure 2B, lanes 7-9) suggesting that E6 has little effect, if any, on STAT1 DNA-binding by IFN- $\gamma$. Supershift assays with antibodies to STAT1 $\alpha$ (p91) (Figure 2B, lanes 12 and 17), STAT2 (p113) (lanes 13 and 18) and PTyr (lanes 14 and 19) identified tyrosine phosphorylated STAT $1 \alpha$ as the component of IFN- $\gamma$ inducible DNAprotein complex.
Induction of ISGF3 or STAT1 DNA-binding by IFNs is normal in cells expressing the HPV-11 E6

Next, we wished to examine whether inhibition of ISGF3 DNA-binding by E6 differs among the benign and transforming subtypes of HPV. To address this question, HT1080 cells were transfected with the HPV11 E6 gene and the neomycin resistant gene. Thirty clones were isolated and analysed for expression of HPV-11 E6 by Northern blotting (data not shown). One of the clones with the highest HPV-11 E6 RNA levels was chosen for further analysis. Stimulation with IFN $-\alpha$ resulted in induction of ISGF3 DNA-binding, which was equal to HT1080 control cells (Figure 3A, compare lanes 2-5 with 7-9). In addition, an equal induction of STAT1 DNA-binding was observed when HT1080 control cells and cells expressing HPV-11 E6 were treated with IFN- $\gamma$ (Figure 2B, lanes 2-4 and 79). Similar data were obtained when HT1080 polyclonal populations expressing HPV-11 E6 were used (data not shown). These findings suggest that inhibition of ISGF3 DNA-binding is not a property of the 'benign' HPV-11 E6.

\section{HPV-18 E6 decreases transactivation of IFN-inducible} genes

Next we examined whether the inhibition of ISGF3 DNA-binding by HPV-18 E6 (Figure 1) had had an effect on transactivation of IFN-inducible genes. To this end, control and HPV-18 E6 expressing HT1080 cells were left untreated (lanes 1 and 5) or treated with IFN- $\alpha$ for 2 (lanes 2 and 6), 4 (lanes 3 and 7) and $6 \mathrm{~h}$ (lanes 4 and 8). RNA was subjected to Northern blot analysis (Figure 4A) using ${ }^{32} \mathrm{P}$-labeled cDNAs of the IFN-inducible genes ISG-15 (top panel) and IFP-53 (middle panel) as probes. Equal loading was verified by probing with glyceraldehyde-

(A)
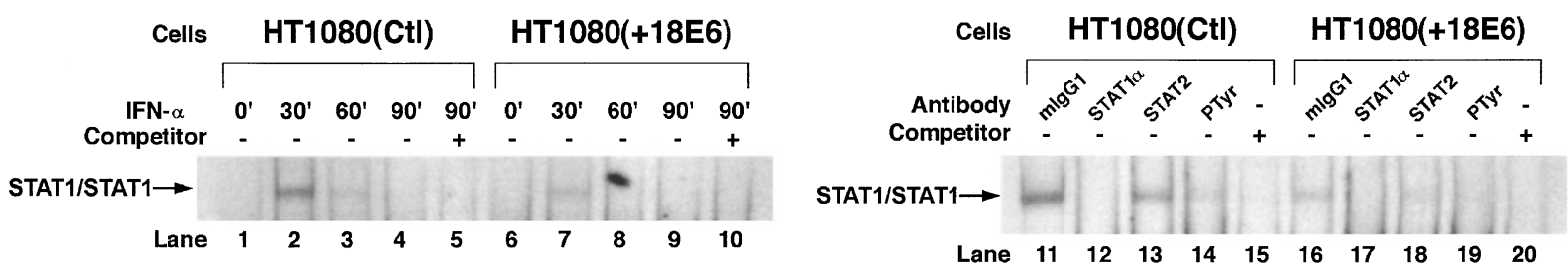

(B)
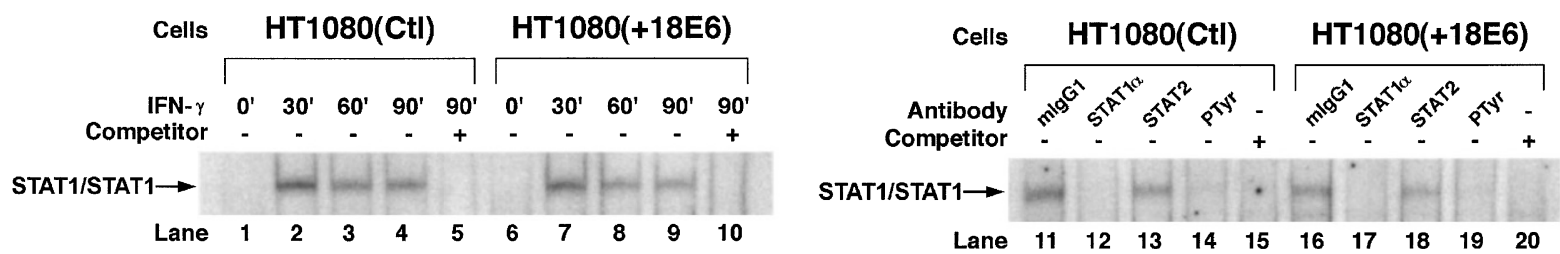

Figure 2 Inhibition of STAT1 DNA-binding by HPV-18 E6 by IFN- $\alpha$ but not IFN- $\gamma$. HT1080 control cells (lanes $1-5$ and $11-15$ ) and HPV-18 E6-expressing HT1080 cells (lanes 6-10 and 16-20) were treated either with $1000 \mathrm{IU} / \mathrm{ml}$ of IFN- $\alpha$ (A) or 500 IU/ml IFN- $\gamma$ (B) for 30 (lanes 2 and 7), 60 (lanes 3 and 8) and 90 min (lanes 4 and 9). Cell extracts were then analysed for DNA-binding using a ${ }^{32}$ P-labeled dsDNA GAS oligonucleotide from IFP-53 gene promoter. A 100-excess of unlabeled dsDNA oligonucleotide was added in the cold competition reactions (A and $\mathbf{B}$, lanes 5, 10, 15 and 20). For supershift assays, extracts from 30 min stimulation either with IFN- $\alpha(\mathbf{A})$ or IFN- $\gamma(\mathbf{B})$ were pre-incubated with $2 \mu \mathrm{g}$ of mouse $\operatorname{IgG}_{1}$ (lanes 11 and 16), anti-STAT1 $\alpha$ (lanes 12 and 17), anti-STAT2 (lanes 13 and 18) and anti-PTyr (lanes 14 and 19) antibodies 
(A)

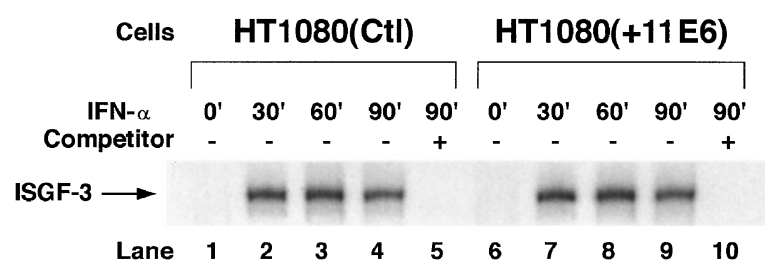

(B)

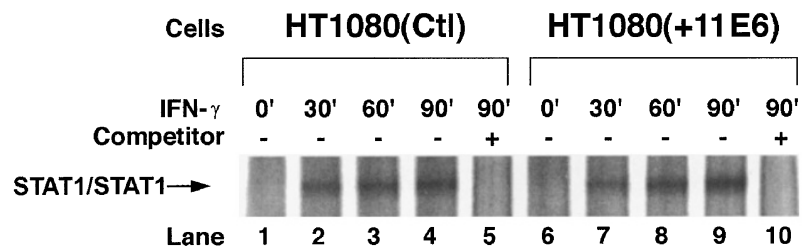

Figure 3 DNA-binding of ISGF3 or STAT1 is not affected by HPV-11 E6. HT1080 control cells (lanes 1-5) and HPV-11 E6expressing HT1080 cells (lanes 6-10) were treated either with $1000 \mathrm{IU} / \mathrm{ml}$ of IFN- $\alpha$ (A) or $500 \mathrm{IU} / \mathrm{ml} \mathrm{IFN-} \gamma(\mathbf{B})$ for 30 (lanes 2 and 7), 60 (lanes 3 and 8) and 90 min (lanes 4 and 9). Cell extracts were then analysed for DNA-binding using either the ${ }^{32} \mathrm{P}$ labeled ISG-15-ISRE dsDNA oligonucleotide (A) or the ${ }^{32} \mathrm{P}$ labeled dsDNA IFP-53-GAS oligonucleotide (B). A 100-excess of either unlabeled dsDNA oligonucleotide was added in the cold competition reactions (A and $\mathbf{B}$, lanes 5 and 10 )

3-phosphate dehydrogenase (GAPDH) ${ }^{32} \mathrm{P}$-labeled cDNA (bottom panel). These experiments showed a diminished IFN- $\alpha$-mediated induction of both ISG-15 and IFP-53 RNA expression in HPV-18 E6 expressing cells compared to control cells (Figure 4A, left panel, compare lane 2 with 6,3 with 7 and 4 with 8). Quantification of the radioactive bands revealed a $\sim 50 \%$ and $\sim 80 \%$ inhibition of $I S G-15$ and IFP-53 gene transactivation respectively by HPV-18 E6 (Figure 4, right panel).

To further verify the above findings, we performed transient transfection assays using the luciferase reporter gene under the control of the ISRE from the promoter of the $I S G-15$ gene (Pine et al., 1994). Induction of luciferase activity was then assessed in both cell lines after stimulation with IFN- $\alpha$ (Figure 4B). Five independent experiments revealed a $30 \%$ inhibition of ISG-15 promoter transactivation by HPV18 E6 in response to IFN- $\alpha$, and this effect was statistically significant $(P<0.05)$. Similar data were obtained when polyclonal populations of HPV-18 E6 expressing HT1080 cells were used in the transient transfections (data not shown). These results are consistent with the decreased levels of ISG-15 RNA expression in Figure $4 \mathrm{~A}$ and demonstrate the negative effect of HPV-18 E6 on IFN- $\alpha$-induced gene transactivation.

\section{IFN- $\alpha$-induced tyrosine phosphorylation of Tyk2 and} STAT2 is decreased in cells expressing HPV-18 E6

Since ISGF3 DNA-binding requires tyrosine phosphorylation of STAT1 and STAT2, we reasoned that tyrosine phosphorylation of these proteins might be affected by HPV-18 E6. To this end, STAT2 phosphorylation (Figure 5A) was examined by a combination of immunoprecipitation with an antiSTAT2 specific antibody and immunoblotting with anti-Ptyr antibodies before (lanes 1 and 4) and after stimulation with IFN- $\alpha$ (lanes 2, 3, 5 and 6). These experiments showed that IFN- $\alpha$-induced tyrosine phosphorylation of STAT2 (Figure 5A, upper panel), when normalized to STAT2 protein levels by immunoblotting (lower panel), was $\sim$ fivefold less in cells expressing the HPV-18 E6 compared to control cells (compare lane 2 with 5 and 3 with 6). Similar observations were made for tyrosine phosphorylation of STAT1 (data not shown).

Since STAT2 phosphorylation requires activation of Tyk2, we then sought to examine whether Tyk2 phosphorylation is affected by HPV-18 E6. Immunoprecipitation of Tyk2 from IFN-induced cell extracts and immunoblotting with anti-Ptyr antibodies (Figure $5 \mathrm{~B}$, top panel) revealed that tyrosine phosphorylation of Tyk 2 was decreased $\sim$ fivefold in cells expressing HPV-18 E6 compared to control cells (compare lane 2 with 5 and 3 with 6 ). In these experiments, the amount of immunoprecipitated Tyk2 was equal between the two cell lines as judged by immunoblot analysis (Figure $5 \mathrm{~B}$, bottom panel). This data suggests that Tyk2 activation is impaired in HPV-18 E6 cells and this may account for the defective tyrosine phosphorylation of STAT2.

\section{Interaction of Tyk2 with GST-E6 fusion proteins}

Considering the ability of E6 to interact with several cellular proteins (reviewed by Rapp and Chen, 1998), we thought that inhibition of Tyk 2 activation might have been due to its interaction with E6. This possibility was further investigated by performing pull down assays of Tyk2 with purified GST-fusion proteins of HPV-11 and HPV-18 E6 (Figure 6). Incubation of cell extracts with the GST-fusion proteins and pull down assays with glutathionesepharose (Figure 6A) revealed an interaction between the endogenous Tyk2 (top panel) and HPV18 E6 (lanes 2 and 3) or HPV-11 E6 (lanes 4 and 5). The interaction between the two proteins was observed even after IFN- $\alpha$ stimulation for $10 \mathrm{~min}$ (lanes 3 and 5). The identity of Tyk 2 protein was verified by comparing Tyk 2 levels in cell extracts from 2fTGH (lane 7) and U1A cells (lane 6), a mutant cell line lacking Tyk2 (Velazquez et al., 1992). These experiments also showed a higher amount of Tyk2 to interact with HPV-18 E6 compared to HPV-11 E6 (compare lanes 2 and 3 with 4 and 5). In addition, IFN- $\alpha$ treatment did not affect the interaction of Tyk 2 with either of the GST-E6 fusion proteins (lanes 3 and 5) indicating that this interaction may take place independently of Tyk 2 activation. It is noteworthy that in similar experiments neither STAT2 nor STAT1 interacted with E6 in vitro (data not shown).

To further analyse the Tyk2/E6 interaction, we examined their association using a wild type (wt) and the catalytically inactive K930I form of Tyk2. To better monitor the interaction, Tyk 2 proteins were overexpressed in HeLa cells using the vaccinia virus/T7 system (Fuerst et al., 1986) (Figure 6B). Pull down assays of wt Tyk2 (lanes 2-4) or Tyk2 K930I mutant (lanes 6-8) with equal amounts of GST-HPV-18 E6 
(lanes 3 and 7) or GST-HPV-11 E6 (lanes 4 and 8) and immunoblot analysis with anti-Tyk 2 antibodies confirmed the Tyk2/E6 interaction and the ability of mutant Tyk2 to co-precipitate with each of the E6 proteins (lanes 7 and 8). In addition, we observed that a higher amount ( fivefold) of Tyk2 co-precipitated with GST-HPV-18 E6 than GST-HPV-11 E6 (compare lane 3 with 4 and 7 with 8 ).

\section{(A)}
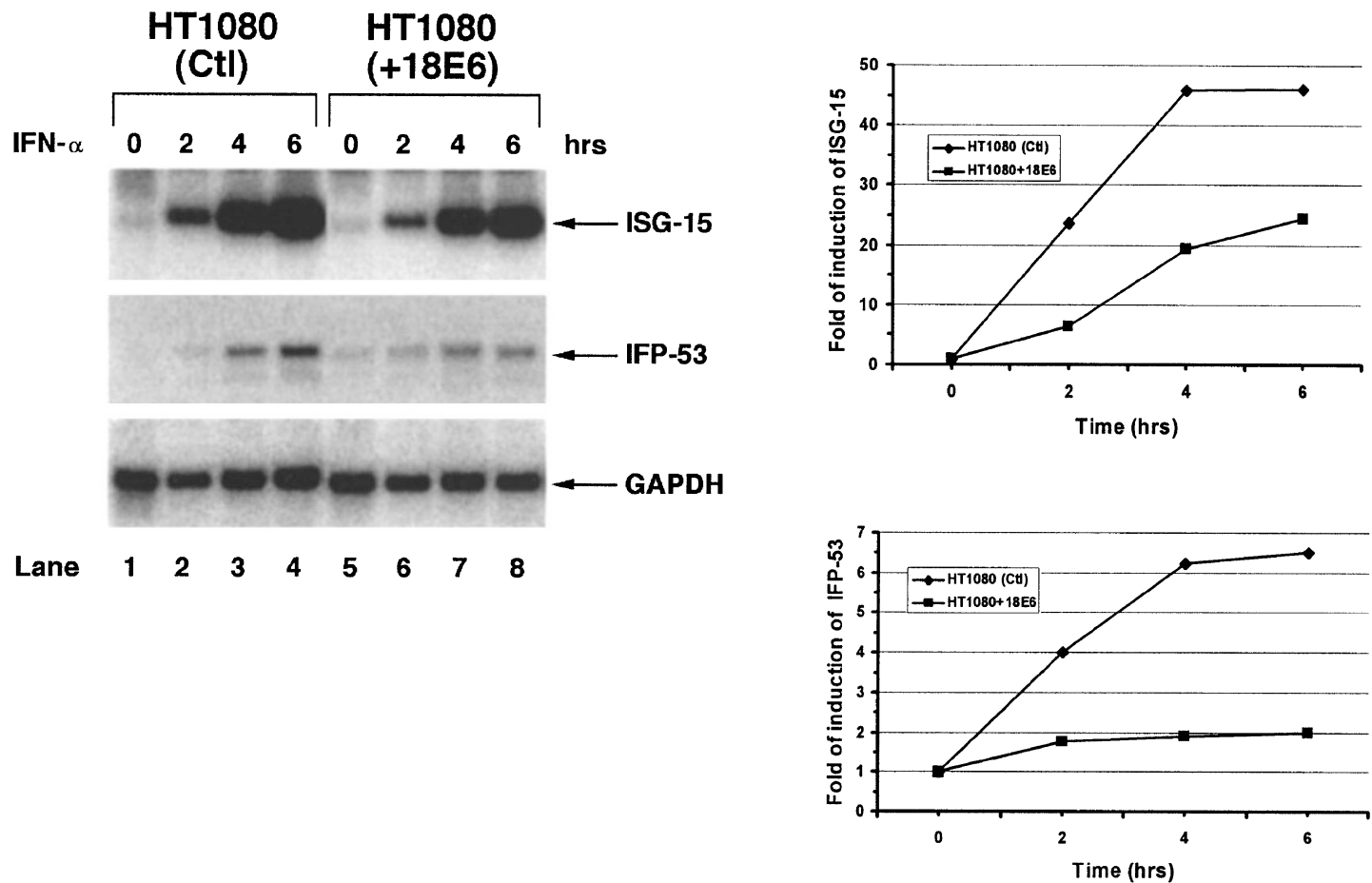

(B)

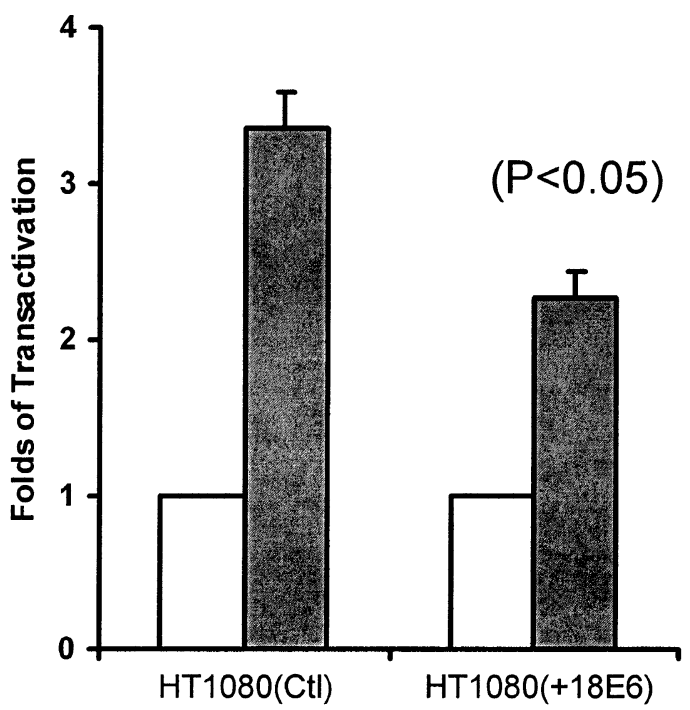

$\square$ None

口 IFNs 
(A)

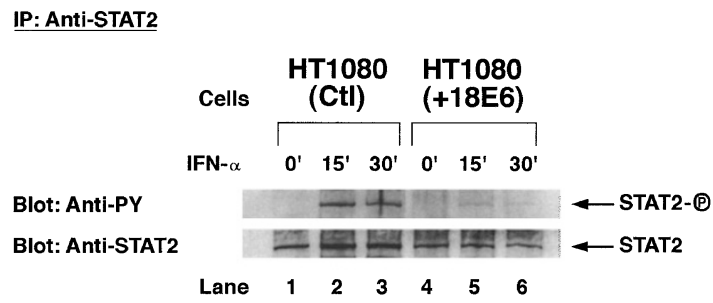

(B)

IP: Anti-Tyk2

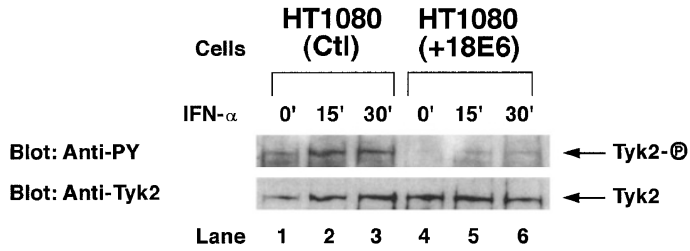

Figure 5 Inhibition of STAT2 and Tyk2 tyrosine phosphorylation by HPV-18 E6. HT1080 control cells (A and B, lanes 1-3) and HT1080 cells expressing HPV-18 E6 (A and B, lanes 4-6) were stimulated with $1000 \mathrm{IU} / \mathrm{ml}$ of $\operatorname{IFN}-\alpha$ (A and $\mathbf{B})$ for 15 (lanes 2 and 3 ) or $30 \mathrm{~min}$ (lanes 3 and 6). Cell extracts $(\sim 500 \mu \mathrm{g}$ ) were used for immunoprecipitation with either anti-STAT2 antibody (A) or anti-Tyk2 antibody (B). Samples were subjected to immunoblotting first with anti-PTyr antibodies (A and $\mathbf{B}$, top panel) followed by immunoblot analysis with anti-STAT2 (A, bottom panel) or anti-Tyk 2 antibody (B, bottom panel)

\section{Co-immunoprecipitation of Tyk2 and E6 proteins}

The Tyk2/E6 interaction was further verified by coimmunoprecipitation. To this end, we used wt Tyk2 and E6 proteins bearing the vesicular stomatitis virus glycoprotein (VSV-G) (Gauzzi et al., 1997) and FLAG epitope in their C-terminus end respectively. VSV-GTyk2 and FLAG-E6 proteins were co-transfected in HeLa cells using the vaccinia virus/T7 system and their expression levels were detected by immunoblotting with either anti-VSV-G (Figure 7, top row) or anti-FLAG antibodies (second from the top row). In these experiments we noticed that expression of FLAG11E6 was much higher than FLAG-18E6 when equal amounts of DNA were used (Figure 7, second from the top row, compare lanes 2 and 3 with 6 and 7). Equal expression HPV-18 and HPV-11 E6 proteins was achieved only when the amount of transfected FLAG-11E6 DNA was decreased tenfold (lanes 4 and 5). In contrast to E6, no differences in VSV-GTyk2 protein expression levels were observed (Figure 7, top row).

The interaction of VSV-G-Tyk2 with FLAG-E6 proteins was then monitored by immunoprecipitation with anti-VSV-G antibodies followed by immunoblot analysis with anti-VSV-G (third row) and anti-FLAG antibodies (bottom row). These experiments demonstrated that Tyk2 interacts with both types of E6 (third and bottom rows, compare lanes 3 and 7). However, the interaction of Tyk2 with HPV-11 E6 was not detected (bottom row, lane 5) upon conditions that expression of FLAG-18E6 was equal to FLAG-11E6 (compare lanes 3 and 5) demonstrating that HPV-18 E6 binds to Tyk2 with a higher affinity than HPV-11 E6. Immunoblot analysis with anti-VSV-G antibodies
(A)

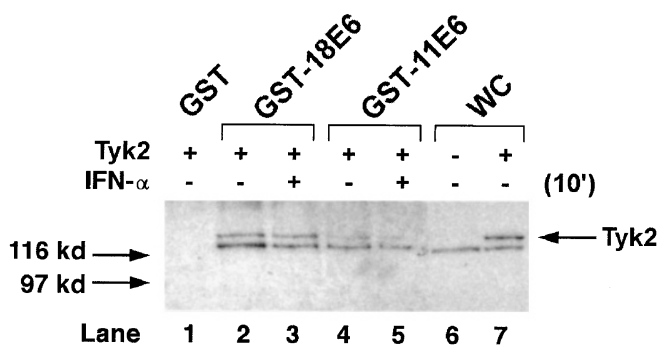

(B)

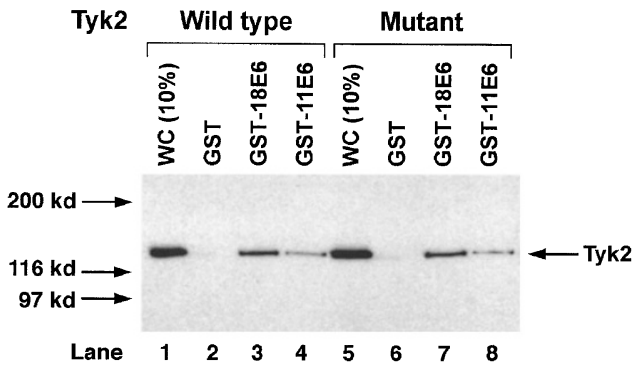

Figure 6 Interaction of Tyk2 with E6 in vitro. (A) Cell extracts (500 $\mu \mathrm{g}$ of protein) of HT1080 cells before (lanes 1, 2 and 4) and after $10 \mathrm{~min}$ stimulation with $1000 \mathrm{IU} / \mathrm{ml}$ of IFN- $\alpha$ (lanes 3 and 5) were mixed with $1 \mu \mathrm{g}$ of purified GST alone (lane 1) or GSTE6 fusion proteins from HPV-18 (lanes 2 and 3) and HPV-11 (lanes 4 and 5). Extracts were pulled down with glutathionesepharose beads and subjected to immunoblot analysis with antibodies to Tyk2. Lanes 6 and 7, $50 \mu \mathrm{g}$ of protein extracts from $\mathrm{U} 1 \mathrm{~A}$ and $2 \mathrm{fTGH}$ cells respectively used as controls to Tyk2 . (B) Wild type Tyk2 (lanes 1-4) or the catalytically inactive Tyk2 K930I mutant (lanes 5-8) was overexpressed in HeLa S3 cells using the vaccinia/T7 system. Cell extracts (100 $\mu \mathrm{g}$ of protein) from each infection/transfection were incubated with $1 \mu \mathrm{g}$ purified GST (lanes 2 and 6), GST-HPV-18 E6 (lanes 3 and 7) or GSTHPV-11 E6 (lanes 4 and 8), pulled down with glutathionesepharose beads and subjected to Western blotting with antibodies against Tyk2. Lanes 1 and 5 represent $10 \%$ of the total input of Tyk2 for each pull-down reaction

showed that an equal amount of VSV-G-Tyk2 was immunoprecipitated in all reactions (third row, lanes 3, 5 and 7 ).

The HPV-18 E6/Tyk 2 interaction requires the $\mathrm{JH}_{6}-\mathrm{JH}_{7}$ domains of $T y k 2$

To better understand the molecular basis of Tyk2/E6 interaction, we then sought to identify the domain(s) of Tyk2 required for binding to E6 (Figure 8). The approach taken was to use either full length VSV-GTyk2 (lanes 1 and 2), a VSV-G-Tyk2 truncated protein consisting of the N-terminus part of Tyk2 only (lanes 3 and 4) and VSV-G-Tyk2 proteins lacking either the first 287 (lanes 5 and 6) or 496 amino acids (lanes 7 and 8) from the N-terminus. These VSV-G-Tyk2 forms were overexpressed in HeLa cells by the vaccinia virus/ T7 system and extracts were used in pull down assays with purified GST-HPV-18 E6 fusion protein (Figure 8A). These experiments showed that the first 287 amino acids of Tyk2, which encompass the $\mathrm{JH}_{6}$ and $\mathrm{JH}_{7}$ domains of Tyk2, are required for the interaction with E6.

The Jaks have been implicated in binding to the cytoplasmic portions of IFN receptors (Colamonici et al., 1994a,b; Kohlhuber et al., 1997) through their $\mathrm{JH}_{6}$ - 


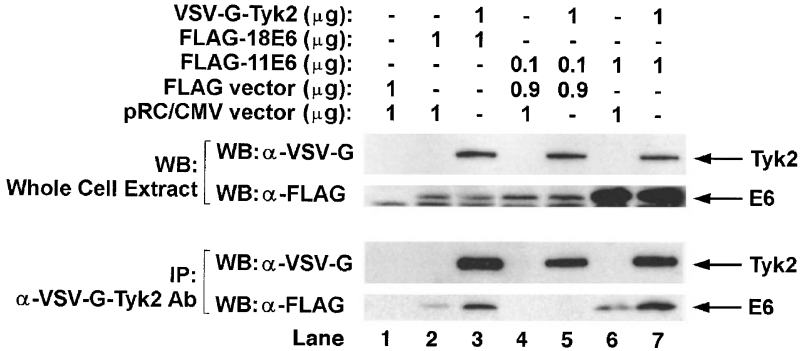

Figure 7 Co-immunoprecipitation of Tyk2 and E6 proteins. FLAG-18E6 (lanes 2 and 3) or FLAG-11E6 protein (lanes, 4, 5, 6 and 7) was expressed either alone (lanes 2, 4 and 6) or together with VSV-G-Tyk2 (lanes 3, 5 and 7) in HeLa cells using the vaccinia virus/T7 system. Expression of E6 and Tyk2 proteins was first detected by immunoblot analysis of $50 \mu \mathrm{g}$ of whole cell extracts with either anti-VSV-G (top row) or anti-FLAG mouse monoclonal antibody (second row). Tyk2/E6 interaction was detected by immunoprecipitation of $1 \mathrm{mg}$ of protein extracts with $2.5 \mu \mathrm{g}$ a mouse monoclonal anti-VSV-G antibody followed by immunoblot analysis with either anti-VSV-G (third row) or antiFLAG monoclonal antibody (bottom row). As control mock transfected HeLa cells were used (lane 1). The amounts $(\mu \mathrm{g})$ of transfected DNA for each of the vectors are indicated on the top

$\mathrm{JH}_{7}$ domains (Richter et al., 1998). To confirm this observation, full length VSV-G-Tyk2 and truncated VSV-G-Tyk2 $\Delta(1-287)$ were overexpressed in HeLa cells by vaccinia virus/T7 system. Pull down experiments with a purified GST-fusion protein encompassing amino acids 465-557 from cytoplasmic part of IFN receptor (Colamonici et al., 1994a,b) resulted in interaction with Tyk 2 but not Tyk $2 \Delta(1-287)$ mutant (Figure 8B). This result shows that HPV-18 E6 interacts with the domains of Tyk2 required for binding to IFN receptor. Similar experiments showed the lack of interactions between the E6 proteins and IFNAR1 (data not shown).

\section{Discussion}

In this paper we have examined the effects of E6 proteins on IFN signaling. The approach we have taken was to establish cell lines from the human HT1080 cells stably expressing the E6 gene. HT1080 cells were derived from a fibrosarcoma but subsequent subcultivation eliminated the growth of fibroblasts and favored proliferation of epithelial cells (Hay et al., 1994). For this reason, HT1080 were shown to be a suitable system to study the functions of E6 protein (Labrecque and Matlashews$\mathrm{ki}$, 1995). Stimulation with IFN- $\alpha$ resulted in inhibition of binding of the ISGF3 complex to the ISRE DNA found in the promoter of the IFNinducible genes (Figure 1A). This effect appeared to be specific for ISGF3, since DNA-binding of other transcription factors induced by IFN- $\alpha$ (i.e. IRF-1, IRF-2) was not modified by HPV-18 E6 (data not shown). In addition, induction of ISGF3 DNAbinding by IFN- $\alpha$ was not modified by the presence of HPV-11 E6 (Figure 3) suggesting that inhibition of Jak-STAT activation is a property of the 'malignant' HPV-18 E6. In contrast to IFN- $\alpha$, induction of STAT1 DNA-binding (Figure 2B) and ISG RNA expression (data not shown) by IFN- $\gamma$
(A)

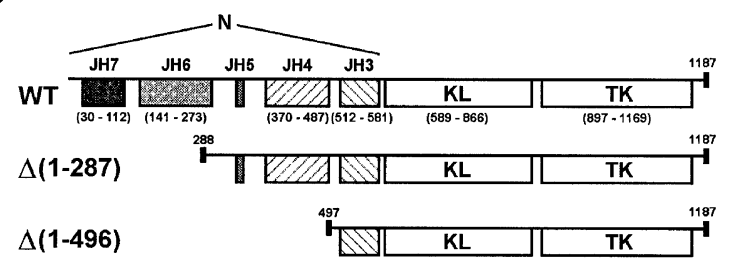

Tyk2
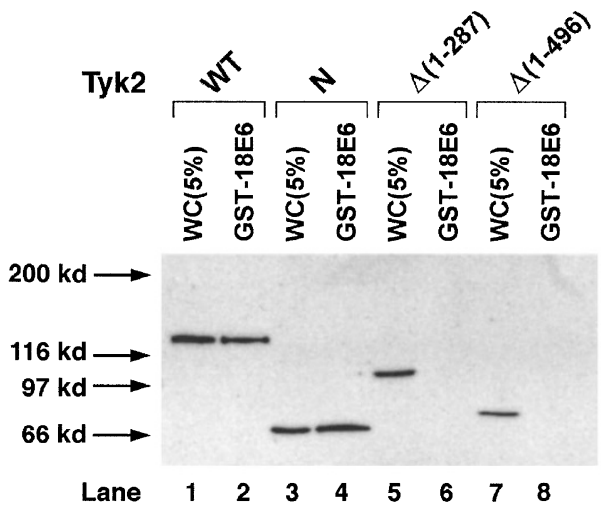

(B)

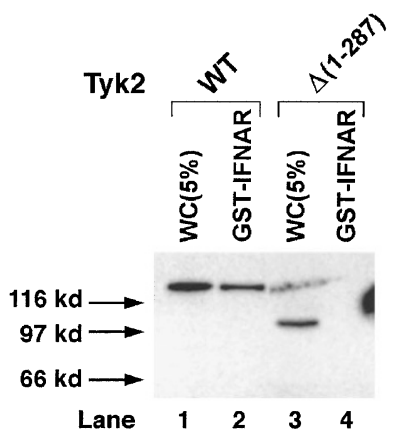

Figure 8 Mapping of Tyk2 interaction domains with E6. (A) Full-length wild type VSV-G-Tyk2 (lanes 1 and 2), N-terminal domain of VSV-G-Tyk2 (lanes 3 and 4) and truncated VSV-GTyk2 lacking either the first 287 [ $\Delta(1-287)$, lanes 5 and 6] or the first $496[\Delta(1-4960)$, lanes 7 and 8$]$ amino acids were expressed in HeLa S3 cells by the vaccinia/T7 system. Cell extracts (100 $\mu \mathrm{g}$ of protein) were used for pull down with $1 \mu \mathrm{g}$ of purified GST-HPV18 E6 (lanes 2, 4, 6 and 8). Samples were subjected to immunoblot analysis with anti-VSV-G antibody. As control, 5\% of the total input for each Tyk2 pull-down reaction was used (lanes 1, 3, 5 and 7). (B) In vitro interaction of Tyk2 with IFNAR1. Full length wild type VSV-G-Tyk2 (lane 2) or $\Delta(1-$ 287) VSV-G-Tyk2 expressed by vaccinia/T7 in HeLa S3 cells $(100 \mu \mathrm{g}$ of protein extracts) were used for pull down with $1 \mu \mathrm{g}$ of purified GST-IFNAR1 (aa 465-557) fusion protein. Samples were analysed by immunoblotting using anti-VSV-G antibody. Lanes 1 and 3 represent $5 \%$ of the total input for each Tyk 2 pulldown reaction

were not affected by HPV-18 E6 suggesting a specific role for HPV-18 E6 in type I IFN signaling.

Phosphorylation of human STAT1 on tyrosine ${ }^{701}$ and human STAT2 on tyrosine ${ }^{690}$ are necessary and sufficient for binding to DNA as part of the ISGF3 complex (Darnell, 1997; Stark et al., 1998). Immunoblot analyses with anti-PTyr antibodies demonstrated a diminished phosphorylation of STAT2 (Figure 5A) and Tyk2 (Figure 5B) by HPV-18 E6 in response to IFN- $\alpha$. We also noticed some differences in the levels of inhibition of ISGF3 DNA-binding and transactivation by the HPV-18 E6. Specifically, IFN- $\alpha$ stimulation 
(A)

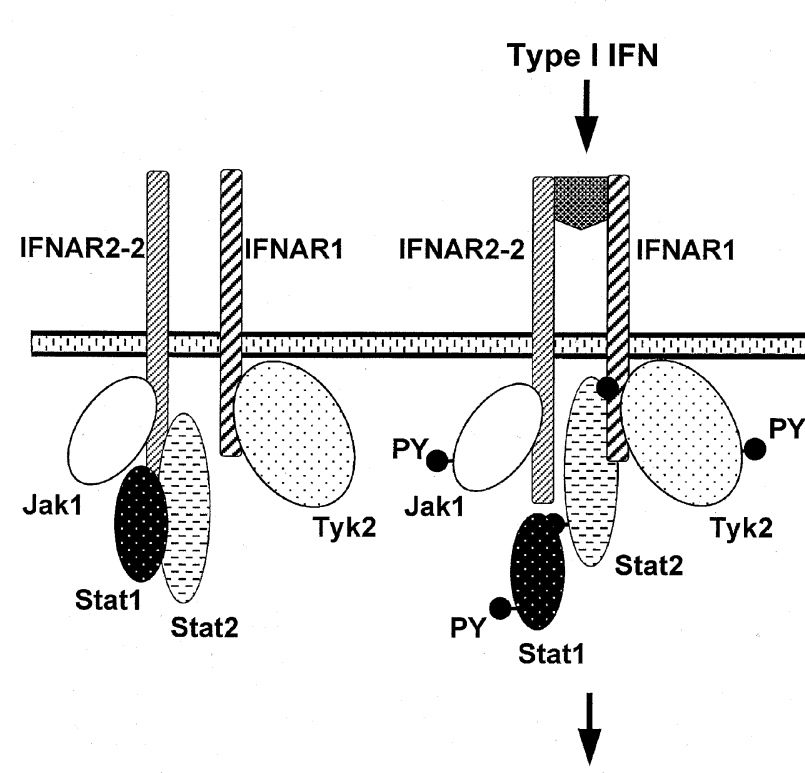

(C)

Type I IFN

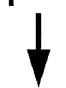

Figure 9 A possible role of Tyk2/E6 interaction in Jak-STAT activation by IFN- $\alpha$. (A) Signaling components are preassociated with the receptor subunits, that is Tyk2 with IFNAR1 and Jak1, STAT1 and STAT2 with IFNAR2-2. (B) IFN- $\alpha$ brings the receptor subunits in proximity, along with the associated Jaks and STATs. Phosphorylation of IFNAR1 on tyrosine ${ }^{466}$ by activated Tyk2 results in binding of the SH2 domain of STAT2 to IFNAR1. STAT2 is then phosphorylated on tyrosine ${ }^{690}$ thus providing a docking site for the SH2 domain of STAT1. STAT1 is consequently phosphorylated on tyrosine ${ }^{701}$. (C) HPV-18 E6 binds to Tyk2 and prevents the association of Tyk2 with IFNAR1. As a result tyrosine phosphorylation of STAT2 and eventually of STAT1 do not take place after binding of IFN- $\alpha$ to the receptor subunits

resulted in $\sim$ fivefold inhibition of ISGF3 DNAbinding but in a modest $\sim 50-80 \%$ inhibition of ISG expression (Figure 4). A possible explanation for this difference may lie within the transactivation capacity of E6, which is mediated through interactions with the basal transcriptional machinery (Rapp and Chen, 1998).

We also detected an interaction between E6 proteins and Tyk2. In regard to it, we found that Tyk2 interacts with a larger fraction of HPV-18 E6 than HPV-11 E6 (Figures 6 and 7). This is consistent with the ability of HPV-18 E6 but not HPV-11 E6 to inhibit IFN- $\alpha$ signaling in HT1080 cells. The E6/Tyk2 interaction appears to be specific for Tyk2 since neither STAT2 nor STAT1 was bound to E6 (data not shown). In addition, the E6/Tyk2 interaction may not require Tyk2 activation since the catalytically inactive mutant Tyk2 K930I was still capable of interacting with E6 to the same degree as wt Tyk2 (Figure 6B). Binding of E6 proteins to Tyk 2 requires the $\mathrm{JH}_{6}-\mathrm{JH}_{7}$ domains of Tyk2 (Figure 8A), which are also important for Tyk2 interaction with IFNAR1 (Figure 8B). Pull down experiments demonstrated that an equal fraction of Tyk 2 (5\% of the total input) interacts with both HPV18 E6 and IFNAR1 (Figure 8).

Work in many laboratories for the past several years has led to a sequential activation model for IFN- $\alpha$ signaling (Pellegrini and Dusanter-Fourt, 1997). The IFN- $\alpha$ receptor is present at low numbers on the surface of all cell types and consists of two transmembrane proteins named IFNAR1 and IFNAR2-2 (Novick et al., 1994; Uze et al., 1990). The IFNAR2 gene generates several alternatively spliced forms, but only the product harboring a long intracytoplasmic domain (IFNAR2-2) is part of a functional IFN- $\alpha$ receptor (Domanski et al., 1995; Lutfalla et al., 1995) (Figure 9). A membrane-proximal 33 amino acid sequence of the cytoplasmic domain of IFNAR1 physically associated with Tyk2 (Colamonici et al., 1994a,b) (Figure 9A). In response to IFN- $\alpha$, Jak1 and Tyk2 become activated by phosphorylation resulting in the phosphorylation of tyrosine ${ }^{466}$ of IFNAR1, which is utilized as a docking site by the SH2 domain of STAT2 (Li et al., 1997; Yan et al., 1996) (Figure 9B). Bound STAT2 to IFNAR1 serves as a substrate of activated Tyk2 resulting in STAT2 phosphorylation on tyrosine ${ }^{690}$, which then provides a docking site for the SH2 domain of STAT1, allowing its tyrosine ${ }^{701}$ to become phosphorylated (Greenlund et al., 1994; Improta et al., 1994) (Figure 9B). According to this model, our findings may be explained by the ability of E6 to interact with Tyk2 and prevent binding of Tyk2 to IFNAR1 (Figure 9C). Binding of E6 to Tyk2 may result in an impaired phosphorylation of IFNAR1 on tyrosine ${ }^{466}$ and sequentially, in inhibition of STAT2 and STAT1 phosphorylations. There are three lines of evidence that favor this possibility. First, cell fractionation experiments have also shown a cytoplasmic localization of E6 proteins (Rapp and Chen, 1998). Second, the $\mathrm{JH}_{6}-\mathrm{JH}_{7}$ domains of Tyk2 are also required for the interaction with the cytoplasmic portion of the IFNAR1 subunit (Figure 8B). Interestingly, the $\mathrm{JH}_{6}-\mathrm{JH}_{7}$ domains are required for efficient phosphorylation of IFNAR1 in vitro and for rendering the kinase activable by IFN- $\alpha$ (Gauzzi et al., 1997). Third, tyrosine phosphorylation of Tyk2 is diminished in cells expressing HPV-18 E6 after stimulation with IFN- $\alpha$ (Figure 5A). Although such a mechanism could be explained by a direct association between Tyk 2 and E6 in vitro, the possibility that the function of Tyk2/E6 
interaction is modulated by other cellular proteins in vivo can not be excluded. Also, the Tyk2/E6 interaction may not result in the destabilization of IFNAR1 since IFNAR1 levels are not affected by the expression of HPV-18 E6 (data not shown).

It should be noted, however, that inhibition of Tyk2 activation by HPV-18 E6 may proceed through more than one but not mutually exclusive mechanisms. In addition to Tyk2/E6 interaction, E6 may also modulate the expression and/or activity of a phosphatase that targets Tyk2 activation (David et al., 1996; You et al., 1999). It is also conceivable that E6 might be able to regulate the expression and/or activity of a specific inhibitor(s) of Tyk2 and/or STAT2. Such inhibitors to the components of the Jak-STAT pathway have been identified and characterized (Starr and Hilton, 1999).

In regard to Tyk2/E6 interactions, E6 binding to Tyk2 took place when GST-fusion proteins with either HPV-18 or HPV-16 E6 were used (data not shown). Also, HPV-16 E6 but not HPV-18 E6 interacted with Jak2 in vitro (data not shown), which may account for the specificity of HPV-18 E6 for IFN- $\alpha$ signaling described in this paper. This is consistent with other findings suggesting that the specificity of Jak kinases may lie in their structural interaction with the IFN receptor and different proteins rather than in the substrate specificity of their kinase domains (Pellegrini and Dusanter-Fourt, 1997). Such differences of E6 function among the 'high risk' HPV subtypes are not unusual. For example, HPV-16 E6 interacts very strongly with IRF-3 in vitro whereas HPV-18 E6 interacts only modestly with IRF-3 (Ronco et al., 1998).

A recent study with the 'high risk' HPV-16 E6 led to the identification of the conserved binding motif E-L$\mathrm{L} / \mathrm{V}-\mathrm{G}$ within the E6 interacting proteins E6AP (a ubiquitin protein ligase) and E6BP (a putative calcium binding protein), which is required for interaction with HPV-16 E6 (Elston et al., 1998). Analysis of the amino acid sequences of Tyk2 identified the presence of a similar sequence E-S-L-G within the JH6 domain of Tyk2 supporting our data that this domain is required for E6/Tyk2 interaction (Figure 8). In regard to E6 protein, the structural features necessary for interaction with Tyk2 are not as yet known. E6 is a small basic protein of 151 amino acids whose major structural characteristic is the presence of two hypothetical zinc fingers. At the base of each of them are two motifs containing cysteines (Cys-X-X-Cys), which are conserved throughout all HPV types (Howley, 1996; Tommasino and Crawford, 1995). This structural characteristic of E6 may be necessary but not sufficient for the specificity of Tyk2/E6 interaction since HPV-18 and HPV-11 E6 proteins, which both contain the zinc finger structures, bind with different affinity to Tyk2 (Figures 6 and 7). The 'high risk' HPV E6 has been shown to bind to the tumor suppressor p53 resulting in p53 inactivation by degradation through the ubiquitin pathway (Rapp and Chen, 1998). Unlike p53, incubation of rabbit reticulocyte lysates containing HPV-18 E6 and Tyk2, STAT1, or STAT2 did not result in degradation of these proteins (data not shown). In addition, Jak-STAT activation was not affected by the expression of a temperature sensitive mutant of p53 in HT1080 cells (data not shown) suggesting that the effect of E6 on Jak-STAT activation is most likely independent of p53 inactivation.

Considering the physiological significance of our findings, the inhibition of Jak-STAT pathway by HPV18 E6 protein may represent one of the mechanisms utilized by HPVs to block IFN action. Additional mechanisms are likely to exist and proceed through the other major HPV oncoproteins. For example, E7 displays considerable structural and functional similarities to adenovirus E1A protein (Howley, 1996; Tommasino and Crawford, 1995). E1A functions as a potent suppressor of IFN signaling (Gutch and Reich, 1991; Kalvakolanu et al., 1991; Leonard and Sen, 1996, 1997; Routes et al., 1996) by interacting with and impairing the transcriptional function of STAT1 (Look et al., 1998). A systematic approach to investigate the regulation of IFN signaling by the HPV oncoproteins may prove helpful to better understand the molecular basis of HPV-resistance to IFN action and may lead to the development of new strategies to combat HPVinfection and associated diseases.

\section{Materials and methods}

\section{Cell culture and transfection}

The human epithelial-like cell line HT1080 (ATCC CCL-121) and HeLa S3 (ATCC CCL-2.2) were maintained in Dulbecco's modified Eagle's medium (DMEM) (Gibco) with $10 \%$ fetal bovine serum (FBS) (Gibco), $2 \mathrm{~mm}$ L-glutamine (Gibco) and 100 units/ml penicillin-streptomycin (Gibco) The HT1080 cells expressing the E6 proteins were established as previously described (Labrecque and Matlashewski, 1995).

\section{Protein expression with the vaccinia/T7 system}

One day before transfection, $0.8 \times 10^{6} \mathrm{HeLa} \mathrm{S3}$ cells were seeded in $6-\mathrm{cm}$ plates. One hour before transfection, the cells were infected with recombinant vaccinia virus containing the bacterial T7/RNA polymerase gene (Fuerst et al., 1986). Transfection was performed using $2 \mu \mathrm{g}$ of plasmid DNA mixed with $5 \mu \mathrm{g}$ Lipofectamine (Gibco, BRL) per plate and cells were incubated in serum-free media at $37^{\circ} \mathrm{C}$ for $5 \mathrm{~h}$. Subsequently, complete DMEM supplied with serum was added to continue incubation for an additional $16 \mathrm{~h}$ before harvesting.

\section{Electrophoretic mobility shift assays (EMSA)}

For the EMSAs the following synthetic double-stranded oligonucleotides (Sheldon Biotech. Center, Montreal) were used: the ISRE of the IFN- $\alpha$-inducible ISG-15 gene (5' - GATCGGGAAAGGGAAACCGAAACTGAAGCC - 3'; ISRE is underlined) (Reich and Darnell, 1989) and the GAS of the IFN- $\gamma$-inducible IFP-53 gene (5'-GATCCAGATTCTCAGAAA-3'; GAS is underlined) (Strehlow et al., 1993). Preparation of whole cell extracts, DNA-binding and supershift assays performed as previously described (Wong et al., 1997). Quantification of the radioactive bands was performed in the linear range of exposure by densitometric analysis using the NIH Image 1.54 software.

\section{Northern blot analysis}

The total RNA was isolated by the guanidinium thiocyanate method (Sambrook et al., 1989). Fifteen $\mu \mathrm{g}$ of RNA was denatured with glyoxal an dimethylsulfoxide and subjected to electrophoresis on a $1.2 \%$ agarose gel at $3 \mathrm{~V} / \mathrm{cm}$ in $10 \mathrm{~mm}$ sodium phosphate buffer pH 7.0 (Sambrook et al., 1989). 
RNA was then transferred onto a nylon membrane (BioTans, ICN) and probed with $\alpha^{32} \mathrm{P}-\mathrm{dATP}$-labeled random primed cDNA probes $\left(5 \times 10^{6}\right.$ c.p.m./ml) (Sambrook et al., 1989). The radioactive bands were visualized by autoradiography and quantified in the linear range of exposure by densitometry using the NIH Image 1.54 software.

\section{Immunoprecipitation and Western blot analysis}

Protein extraction, immunoprecipitation and immunoblot analysis were performed as previously described (Wong et al., 1997). For immunoblot analyses the following antibodies were used: (i) anti-STAT1 $\alpha$ (Santa Cruz Biotechnology); (ii) anti-STAT2 (Upstate Biotech. Inc.); (iii) anti-Tyk2 (Santa Cruz Biotech); (iv) anti-PTyr (Santa Cruz Biotech.) antibodies; (v) anti-VSV-G antibodies (Boehringer Mannheim); (vi) anti-FLAG antibodies (Sigma). After incubation with anti-mouse or anti-rabbit peroxidase-conjugated antibody ( $1: 1000$ dilution; Amersham Corp.), proteins were visualized using the enhanced chemiluminescence (ECL) detection system (Amersham Corp.) according to manufacturer's instructions. Quantification of the bands in the linear range of exposure was performed by densitometry using the NIH Image 1.54 software.

\section{GST pull-down and interaction assays}

GST-E6 fusion proteins were prepared from bacterial cultures induced with $0.1 \mathrm{~mm}$ isopropyl- $\beta$-D-thiogalactopyranoside (IPTG) (Sigma) for $3 \mathrm{~h}$ using the standard protocol (Sambrook et al., 1989). Cell extracts were prepared with a lysis buffer containing $50 \mathrm{mM}$ Tris. $\mathrm{HCl}$ pH 8.0, $120 \mathrm{mM}$ $\mathrm{NaCl}, 0.5 \%$ Nonidet P-40 (ICN), $1 \mathrm{~mm}$ dithiothreitol (DTT), $0.2 \mathrm{mM}$ PMSF, $4 \mu \mathrm{g} / \mathrm{ml}$ aprotinin, $1 \mu \mathrm{g} / \mathrm{ml}$ leupeptin and $1 \mu \mathrm{g} / \mathrm{ml}$ pepstatin. Pull down experiments were carried out with $1 \mu \mathrm{g}$ of purified GST-fusion proteins, $100-500 \mu \mathrm{g}$ of protein extracts and $20 \mu \mathrm{l}$ of packed glutathione sepharose ${ }^{\mathbb{R}}$
4B beads (Pharmacia) at $4^{\circ} \mathrm{C}$ for $2 \mathrm{~h}$. The beads were then washed four times with the above lysis buffer and subjected to SDS-PAGE.

\section{Luciferase assays}

Cells were transfected with the ISG-15-ISRE-linked luciferase reporter plasmid 15KWTtkGL3 DNA (Pine et al., 1994). Thirty-six hours after transfection, cells were treated with $1000 \mathrm{IU} / \mathrm{ml}$ of $\mathrm{IFN}-\alpha / \beta$ for $6 \mathrm{~h}$. Luciferase activity was measured by Lumat LB9501 (Berthold) equipment using the luciferase assay kit (Promega) according to manufacturer's specification. All transfections were normalized to the $\beta$ galactosidase activity.

\section{Acknowledgments}

We thank L Banks for GST-fusion proteins of HPV-16 E6 and helpful suggestions; O Colamonici for the GSTIFNAR 1 fusion protein and antibodies to IFNAR1; R Pine for $15 \mathrm{KWTtkGL} 3$ construct; G Stark for $2 \mathrm{fTGH}$ and U1A cells; T Decker for ISG-15 and IFP-53 cDNAs and useful suggestions. This work has been primarily supported by a grant from The Cancer Research Society (CRS) Inc. to AE Koromilas, and by grants from the Medical Research Council (MRC) of Canada and The National Cancer Institute of Canada (NCIC) to AE Koromilas and $\mathrm{G}$ Matlashewski. Work at the Institute Pasteur was in part supported by a grant to $\mathrm{S}$ Pelligrini by the Association pour la Recherche contre le Cancer. AR Cuddihy is supported by a CRS studentship and AHT Wong by a NCIC scholarship and MC Gauzzi by a fellowship from the Fondation pour la Recherche Medicale. AE Koromilas is a member of the Terry Fox Group of Molecular Oncology and a recipient of an MRC Scientist Award.

\section{References}

Bluyssen HAR, Durbin JE and Levy DE. (1996). Cytokine Growth Factor Rev., 1, 11-17.

Colamonici OR, Yan H, Domanski P, Handa R, Smalley D, Mullersman J, Witte M, Krishnan K and Krolewski J. (1994a). Mol. Cell. Biol., 14, 8133-8142.

Colamonici OR, Uyttendaele H, Domanski P, Yan H and Krolewski JJ. (1994b). J. Biol. Chem., 269, 3518-3522.

Darnell Jr DE. (1997). Science, 277, 1630-1635.

Darnell Jr DE, Kerr IM and Stark GR. (1994). Science, 264, $1415-1421$.

David M, Zhou G, Pine R, Dixon JE and Larner AC. (1996). J. Biol. Chem., 271, $15862-15865$.

Domanski P, Witte M, Kellum M, Rubinstein M, Hackett R, Pitha P and Colamonici OR. (1995). J. Biol. Chem., 270, $21606-21611$.

Elston RC, Napthine S and Doorbar J. (1998). J. Gen. Virol., 79, $371-374$.

Fuerst TR, Niles EG, Studier FW and Moss B. (1986). Proc. Natl. Acad. Sci. USA, 86, 2365-2368.

Gauzzi MC, Barbieri G, Richter MF, Uze G, Ling L, Fellous M and Pellegrini S. (1997). Proc. Natl. Acad. Sci. USA, 94, $11839-11844$.

Greenlund AC, Farrar MA, Viviano BL and Schreiber RD. (1994). EMBO J., 13, 1591-1600.

Gutch MJ and Reich NC. (1991). Proc. Natl. Acad. Sci. USA, 88, 7913-7917.

Hay R, Caputo J, Chen TR, Macy M, McClintoch P and Reid Y. (eds) (1994). ATCC: Cell lines and hybridomas. American Type Culture Collection, 8th edn. p.70.

Howley PM. (1996). In: Fundamental Virology. Bernard DMK, Fields $\mathrm{N}$ and Howley PM, (eds). LippincottRaven: Philadelphia, PA, USA. pp. 2045-2076.

Improta T, Shindler C, Horvath CM, Kerr IM, Stark GR and Darnell Jr JE. (1994). Proc. Natl. Acad. Sci. USA, 91, $4776-4780$.

Kalvakolanu DV, Bandyopadhyay SK, Harter ML and Sen GC. (1991). Proc. Natl. Acad. Sci. USA, 88, $7459-7463$.

Kohlhuber F, Rogers NC, Watling D, Feng J, Guschin D, Briscoe J, Witthuhn BA, Kotenko SV, Pestka S, Stark GR, Ihle JN and Kerr IM. (1997). Mol. Cell. Biol., 17, $695-706$.

Kovarik P, Stoiber D, Novy M and Decker T. (1998). EMBO J., 17, 3660-3668.

Labrecque S and Matlashewski GJ. (1995). Oncogene, 11, $387-392$.

Leonard GT and Sen GC. (1996). Virology, 224, 25-33.

Leonard GT and Sen GC. (1997). J. Virol., 71, 5095-5101.

Li A, Leung S, Kerr IM and Stark GR. (1997). Mol. Cell. Biol., 17, 2048- 2056.

Look DC, Roswit WT, Frick AG, Gris-Alevy Y, Dickhaus DM, Walter MJ and Holtzman MJ. (1998). Immunity, 9, $871-880$.

Lutfalla G, Holland SJ, Cinato E, Monneron D, Reboul J, Rogers NC, Smith JM, Stark GR, Gardiner K, Mogensen KE, Kerr IM and Uze G. (1995). EMBO J., 14, $5100-$ 5108.

Novick D, Cohen B and Rubenstein M. (1994). Cell, 77, $225-234$. 
Pellegrini S and Dusanter-Fourt I. (1997). Eur. J. Biochem., 248, 615-633.

Pine R, Canova A and Schindler C. (1994). EMBO J., 13 $158-167$.

Rapp L and Chen JJ. (1998). Biochim. Biophys. Acta, 1378, F1-F19.

Reich NC and Darnell Jr JE. (1989). Nucleic Acids Res., 17, $3415-3424$.

Richter MD, Dumenil G, Uze G, Fellous M and Pellegrini S. (1998). J. Biol. Chem., 273, 24723 - 24729.

Rockley PF and Tyring SK. (1995). Pharmac. Ther., 65, $265-287$.

Ronco LV, Karpova AY, Vidal M and Howley PM. (1998). Genes Dev., 12, 2061-2072.

Routes JM, Li H, Bayley ST, Ryan S and Klemm DJ. (1996). J. Immunol., 156, 1055-1061.

Sambrook J, Fritsch EF and Maniatis T. (1989). Molecular Cloning: A Laboratory Manual. 2nd edn. Cold Spring Harbor Laboratory Press: Cold Spring Harbor, NY.

Shah KV and Howley PM. (1996). In: Fundamental Virology. Bernard DMK, Fields $\mathrm{N}$ and Howley PM, (eds). Lippincott-Raven: Philadelphia, PA. pp. 20772110.

Sherman L and Schlegel R. (1996). J. Virol., 70, 3269-3279.

Stark GR, Kerr IM, Williams BRG, Silverman RH and Schreiber RD. (1998). Аnnu. Rev. Biochem., 67, $227-$ 264.
Starr R and Hilton DJ. (1999). BioEssays, 21, 47- 52.

Stellato G. (1992). Sex. Transmit. Dis., 19, 124-126.

Strehlow I, Seegert D, Frick C, Bange F-C, Schindler C, Bottger EC and Decker T. (1993). J. Biol. Chem., 268, $16590-16595$.

Tommasino M and Crawford L. (1995). BioEssays, 17, $509-$ 518.

Trofatter KF. (1991). Dermatol. Clin., 9, 343-352.

Uze G, Lutfalla G and Gresser I. (1990). Cell, 60, 225-234.

Velazquez L, Fellous M, Stark GR and Pellegrini S. (1992). Cell, 70, 313-322.

Vilcek J and Sen GC. (1996). In: Fundamental Virology. Bernard DMK, Fields N and Howley PM, (eds). Lippincott-Raven: Philadelphia, PA. pp. 375-400.

Wong AHT, Tam NWN, Yang YL, Cuddihy AR, Li S, Kirchhoff S, Hauser H, Decker T and Koromilas AE. (1997). EMBO J., 16, $1291-1304$.

Yan H, Krishnan K, Greenlund AC, Gupta S, Lim JTE, Schreiber RD, Schindler CW and Krolewski JJ. (1996). EMBO J., 15, $1064-1074$.

You N, Yu D-H and Feng G-S. (1999). Mol. Cell. Biol., 19, $2416-2424$.

zur Hausen H. (1996). Biochim. Biophys. Acta, 1288, F55F78. 